\title{
Diagnóstico precoce das cardiopatias congênitas: uma revisão integrativa
}

\author{
Early diagnosis of congenital heart disease: an integrative review \\ Diagnóstico precoz de la enfermedad cardíaca congénita: Una revisión Integrativa \\ Taynar da Costa Almeida PAVÃO ${ }^{(1)}$ \\ Joseildes Castelo Branco SOUZA ${ }^{(1)}$ \\ Luzinéa de Maria Pastor FRIAS (1) \\ Líscia Divana Carvalho SILVA ${ }^{(1)}$
}

Recebido: 27 out 2016 Revisado: 21 mar 2017 Aceito: 28 nov 2017

\section{Autor de}

correspondência: Líscia Divana Carvalho Silva

liscia@elointernet.com.br

Conflito de interesses: Os autores declaram não haver nenhum interesse profissional ou pessoal que possa gerar conflito de interesses em relação a este manuscrito.

(1) Hospital Universitário da Universidade Federal do Maranhão - HUUFMA, São Luís, MA, Brasil.

\begin{abstract}
Resumo
As cardiopatias congênitas constituem uma das principais causas de morbimortalidade neonatal; possuem manifestações clínicas distintas e necessitam de diagnóstico preciso devido a sua rápida evolução para condições ameaçadoras da vida. O objetivo do estudo foi analisar o diagnóstico precoce das cardiopatias congênitas. Os critérios de inclusão foram os artigos disponíveis na íntegra no portal e nos idiomas português, inglês e espanhol, publicados entre os anos de 2005 a 2015 que retratassem o diagnóstico das cardiopatias congênitas. Utilizou-se as seguintes bases de dados: PUBMED (National Center for Biotechnology Information and National Library of Medicine), BVS (Biblioteca Virtual em Saúde), o portal da Coordenação de Aperfeiçoamento de Pessoal de Ensino Superior (CAPES) e LILACS (Literatura Latino-Americana de Ciências da Saúde). Seguiu-se o modelo descrito por Mendes, Silveira e Galvão (2008), que sugere a divisão da revisão em seis etapas. Os artigos selecionados foram submetidos a uma avaliação de rigor metodológico da Critical Appraisal Skills Programme - CASP. Obteve-se uma amostra de 11 artigos. Os métodos diagnósticos mais frequentemente utilizados e com relevância são a ultrassonografia fetal (translucência nucal) e o ecocardiograma fetal. O rastreamento ultrassonográfico na gestante constitui a melhor forma de detecção das malformações no primeiro trimestre, e o ecocardiograma fetal um método importante utilizado para análise mais acurada das cardiopatias. Outros métodos utilizados nos recém-nascidos são o exame físico criterioso, a oximetria de pulso e o ecocardiograma do recém-nascido. A avaliação do recém-nascido com suspeita de cardiopatia deve ser realizada de maneira criteriosa e rotineira, nessa perspectiva, destaca-se a inclusão de outros profissionais devidamente treinados para a realização da oximetria de pulso arterial (enfermagem) e do ecocardiograma do recém-nascido (neonatologistas). Constata-se a importância do diagnóstico precoce para um tratamento adequado, prevenção de agravos e sequelas oportunizando a melhora do prognóstico e permitindo a sobrevida prolongada à saúde da criança, com ênfase na participação de outros profissionais para a qualidade do resultado final obtido.
\end{abstract}

Descritores: Cardiopatias Congênitas; Diagnóstico Precoce; Diagnóstico Pré-natal. 


\begin{abstract}
Congenital heart diseases are a leading cause of neonatal morbidity and mortality; have distinct clinical manifestations and require accurate diagnosis due to their rapid evolution to life threatening conditions. The objective of this study was to analyze the early diagnosis of congenital heart defects. Inclusion criteria were the articles available in full on the website and in the languages Portuguese, English and Spanish, published between the years of 2005 to 2015 representing the diagnosis of congenital heart defects. We used the following databases: PUBMED (National Center for Biotechnology Information and National Library of Medicine), the VHL (Virtual Health Library), the portal of Coordenação de Aperfeiçoamento de Pessoal de Ensino Superior (CAPES) and LILACS (Latin American literature in health sciences). This was followed by the model described by Mendes, Silveira and Galvão (2008), which suggests the review Division in six steps. Selected articles were submitted to an assessment of methodological rigor of Critical Appraisal Skills Programme-CASP. Obtained a sample of 11 articles. The most frequently used diagnostic methods and relevance are the fetal Ultrasound (nuchal translucency) and fetal echocardiography. The ultrasound tracking in pregnant women is the best way to detect malformations in the first quarter, and the fetal Echocardiogram a important method used for more accurate analysis of the heart. Other methods used in newborns are careful physical examination, pulse oximetry and the echo of the newborn. The evaluation of the newborn with suspected heart disease must be carried out in a careful way and routine, from this point of view, the inclusion of other professionals properly trained to perform the arterial pulse oximetry (nursing) and the echo of the newborn (neonatologists). Notes the importance of early diagnosis for a proper treatment, prevention of diseases and providing opportunities for the improvement of the prognosis sequelae and enabling extended survival to child health, with emphasis on the participation of other professionals for the quality of the final result obtained.
\end{abstract}

Keywords: Heart Defects, Congenital; Early Diagnosis; Prenatal Diagnosis.

\title{
Resumen
}

Enfermedades cardíacas congénitas son una causa importante de morbilidad neonatal y mortalidad; tienen manifestaciones clínicas distintas y requieren un diagnóstico preciso debido a su rápida evolución a las condiciones mortales. El objetivo de este estudio fue analizar el diagnóstico precoz de defectos congénitos del corazón. Criterios de inclusión fueron los artículos disponibles en completa en el sitio web y en los idiomas portugués, Inglés y español, publicados entre los años de 2005 a 2015, que representa el diagnóstico de defectos congénitos del corazón. Utilizamos las siguientes bases de datos: PUBMED (National Center for Biotechnology Information and National Library of Medicine), la BVS (Biblioteca Virtual en Salud), el portal de la Coordenação de Aperfeiçoamento de Pessoal de Ensino Superior (CAPES) y LILACS (literatura latinoamericana en Ciencias de la salud). Esto fue seguido por el modelo descrito por Mendes, Silveira y Galvão (2008), que sugiere la revisión de la división en seis pasos. Artículos seleccionados fueron sometidos a una evaluación del rigor metodológico de valoración crítica, habilidades programa-CASP. Obtener una muestra de 11 artículos. Relevancia los métodos de diagnóstico más utilizados son la ecografía fetal (translucencia nucal) y ecocardiografía fetal. El ultrasonido de seguimiento en mujeres embarazadas es la mejor manera de detectar malformaciones en el primer trimestre y el ecocardiograma fetal un importante método utilizado para el análisis más exacto del corazón. Otros métodos utilizados en los recién nacidos son el examen físico cuidadoso, oximetría de pulso y el eco del recién nacido. . La evaluación del recién nacido con sospecha de enfermedad cardíaca debe realizarse de manera cuidadosa y sistemática, desde este punto de vista, la inclusión de otros profesionales debidamente entrenado para realizar la oximetría de pulso arterial (enfermería) y el eco del recién nacido (neonatólogos). Destaca la importancia del diagnóstico precoz para un tratamiento adecuado, prevención de enfermedades y ofrecer oportunidades para la mejora de las secuelas de pronóstico y permitiendo la supervivencia extendida a la salud del niño, con énfasis en la participación de otros profesionales para la calidad del resultado final obtenido.

Palabras-claves: Cardiopatías Congénitas; Diagnóstico Precoz; Diagnóstico Prenatal. 


\section{Introdução}

ISSN 2179-6750

As cardiopatias congênitas (CC) são uma anormalidade do coração e dos grandes vasos que apresentam grande importância funcional em nascidos vivos, sendo mais frequente em fetos. ${ }^{1,2}$ As CC dividem-se em acianóticas e cianóticas; as primeiras são comunicações intercavitárias, intravasculares, mistas e alterações valvares; já as cianóticas são as que precisam de intervenções terapêuticas imediatas. ${ }^{3,4}$ As malformações cardíacas podem ser isoladas, resultado de alterações genéticas e de medicações de uso materno como anticonvulsivantes e antidepressivos; ${ }^{5,6}$ tem amplo aspecto clínico, são sintomáticas ou assintomáticas e surgem em decorrência da embriogênese defeituosa durante o período gestacional da $3^{a}$ até a $8^{a}$ semana Existe uma associação entre a presença de anomalias congênitas, menor duração da gestação e menor escore no índice de APGAR (Appearance, Pulse, Grimace, Activity, Respiration). As CC apresentam elevada mortalidade no primeiro ano de vida, a prevalência varia dependendo da população estudada e pode atingir $1 \%$ da população pós-natal. ${ }^{1,2,7}$

A incidência de CC varia entre $0,8 \%$ nos países mais desenvolvidos a 1,2\% nos países subdesenvolvidos. ${ }^{7}$ A maioria da população de crianças cardiopatas no Brasil é atendida pelo Sistema Único de Saúde ${ }^{8}$ a prevalência da população brasileira é de 9,58 para cada 1.000 (mil) nascidos vivos. Estima-se que as CC acometem em torno de 0,9\% dos nascidos vivos, sendo de 20 a 30\% com defeitos estruturais graves e destes 3 a $5 \%$ morrem no período neonatal. Em torno de $30 \%$ dos recém-nascidos recebem alta hospitalar sem o diagnóstico, e evoluem para choque, hipóxia ou óbito precoce, antes de receber tratamento adequado. Como o defeito na estrutura e função do coração pode ser desenvolvido até a $8^{a}$ semana de gestação, o diagnóstico deve ser realizado o mais breve possível, ainda durante a gravidez (até a $20^{a}$ semana- $5^{\circ}$ mês)..$^{5,9}$

Nesse sentido, a importância do diagnóstico precoce das cardiopatias congênitas durante o pré-natal, a melhoria da qualidade dos exames de imagem, treinamento de profissionais habilitados na realização da morfologia fetal, a indicação 
fundamentada de exame de ecocardiograma fetal e do recém-nascido além de outros, torna-se relevante. ${ }^{2}$ Gestantes com idade superior a 35 anos merecem uma avaliação prénatal mais rigorosa devido às maiores chances de malformações fetais, fato este que obstetras têm incluído o ecocardiograma fetal entre os exames de rotina nesse grupo de pacientes como complemento a avaliação da ultrassonografia morfológica. ${ }^{5,6,9}$

O período neonatal para o portador de CC pode ser crítico, devido principalmente à gravidade de alguns defeitos e modificações fisiológicas. O exame clínico nessa fase tornase valioso na suspeita clínica de cardiopatia como o sopro cardíaco, cianose, taquipneia e arritmia cardíaca. A radiografia torácica vem perdendo sua importância diagnóstica, devido à dificuldade em obter boas imagens. A oximetria de pulso arterial é um bom método de triagem, podendo ser realizada de rotina em recém-nascidos aparentemente saudáveis com idade gestacional maior que 34 semanas, o que mostra elevada sensibilidade e especificidade para detecção precoce das cardiopatias, apesar de não ter a capacidade de excluir ou confirmar a presença da doença e nem de orientar a conduta inicial frente a um caso positivo. ${ }^{10,11}$ O ecocardiograma fornece informações hemodinâmicas importantes como a estimativa da pressão pulmonar e avaliação da função ventricular, diminuindo as indicações de realização do cateterismo cardíaco. A sensibilidade do exame tem alcançado um valor de segurança de até $83 \%$, pois identifica o risco e oportuniza o tratamento adequado. Entretanto, o ecocardiograma exame é realizado somente em $10 \%$ dos recémnascidos. ${ }^{2,5,10}$ Como na maioria das unidades neonatais, a alta hospitalar é realizada entre 36 e 48 horas de vida, a manifestação clínica da cardiopatia pode ainda não ter ocorrido principalmente naquelas com fluxo sistêmico dependente de canal arterial, além disso, a ausculta cardíaca pode ser aparentemente normal nesta fase. Portanto, a utilização adequada dos métodos diagnósticos, só será plenamente valorizada, se a cardiopatia for inicialmente diagnosticada pelo clínico. ${ }^{2}$

O diagnóstico precoce das CC durante a gestação contribui para um melhor atendimento do recém-nascido e aumenta as chances de sucesso no tratamento. Não 
estão claros na literatura científica os métodos mais utilizados para esse diagnóstico. Assim, com o objetivo de superar tais limitações, esse estudo propõe uma revisão sobre os principais métodos de diagnóstico das cardiopatias congênitas na assertiva de que o diagnóstico precoce torna-se fundamental para a identificação do risco e agravo a saúde.

\section{Metodologia}

Selecionou-se como método de pesquisa para este estudo a revisão integrativa da literatura sobre o diagnóstico precoce das cardiopatias congênitas.

Foi utilizado o modelo descrito por Mendes, ${ }^{11}$ que sugere a divisão da revisão em seis etapas: identificação do tema e seleção da hipótese; estabelecimento de critérios para inclusão e exclusão de estudos/amostragem e busca na literatura; definição das informações a serem extraídas dos estudos selecionados/categorização dos estudos; avaliação dos estudos incluídos; interpretação dos resultados e a apresentação da revisão/síntese do conhecimento.

A primeira etapa consistiu na seleção da hipótese para esclarecimento do problema de pesquisa relacionado ao diagnóstico das cardiopatias congênitas, a partir do seguinte questionamento: Como está sendo realizado o diagnóstico das cardiopatias congênitas? A segunda etapa foi o estabelecimento dos critérios de inclusão e exclusão a fim de nortear a busca e seleção dos artigos. Os critérios de inclusão foram os artigos nacionais e internacionais disponíveis na íntegra no período de 2005 a 2015, período em que houve maior discussão sobre os protocolos definidos. Os critérios de exclusão foram: estudos que retratassem o diagnóstico em outros tipos de cardiopatias ou doenças associadas; as intervenções terapêuticas ou tratamentos complementares utilizados nas cardiopatias congênitas; editoriais; resenhas; monografias; textos não científicos e artigos científicos sem disponibilidade na íntegra. 
Os dados foram coletados entre os meses de setembro e outubro de 2015. Foram utilizadas as seguintes bases de dados: National Center for Biotechnology Information and National Library of Medicine - PUBMED, Biblioteca Virtual em Saúde - BVS, o portal da Coordenação de Aperfeiçoamento de Pessoal de Ensino Superior - CAPES e Literatura Latino-Americana de Ciências da Saúde LILACS. A busca pelas publicações se deu por meio dos Descritores em Saúde - DeCS: cardiopatia congênita (congenital heart disease, enfermedad cardíaca congénita), diagnóstico (diagnostics, diagnóstico), diagnóstico precoce (early dianostic, diagnóstico precoz), diagnóstico pré-natal (prenatal diagnostics, diagnóstico prenata), diagnóstico diferencial (differential diagnostics, diagnóstico diferencia), genética (genetics, genética), avaliação (avaluation, evaluación), cardiologia (cardiology, cardiología). Foram feitos os seguintes cruzamentos de descritores: diagnóstico / cardiopatia congênita (diagnostics / congenital heart disease) diagnóstico precoce / cardiopatias congênitas (early diagnostic / congenital heart disease) e diagnóstico pré-natal / cardiopatia congênita (prenatal diagnostics / congenital heart disease).

Foram encontrados 92 artigos, excluindo-se aqueles que não se enquadravam nos critérios de inclusão e aqueles duplicados nas bases de dados e/ou publicados em duplicidade de idiomas, selecionou-se inicialmente 17 artigos. Quando a questão norteadora e os objetivos dos artigos apresentavam dúvidas, lia-se o artigo na íntegra, em havendo consenso entre os pesquisadores, fez-se a inclusão. Na terceira etapa os artigos selecionados foram submetidos a uma avaliação de rigor metodológico de revisão sistemática da Critical Appraisal Skills Programme - CASP. ${ }^{12}$ Essa avaliação é um escore com dez itens pontuáveis que classifica os artigos. Os artigos com pontuação de 06 a 10 foram classificados como de boa qualidade metodológica e viés reduzido e para os artigos com pontuação mínima de cinco a classificação é de estudo com qualidade metodológica satisfatória, porém com risco de viés aumentado. Nesta etapa foram analisados os 17 artigos selecionados, permanecendo somente aqueles que apresentavam boa qualidade 
metodológica, sendo, portanto, excluídos 06 artigos. A amostra final foi constituída por onze artigos, conforme demonstrado na figura 1, sendo três artigos na base de dados PUBMED, quatro artigos na BVS, dois artigos CAPES e dois no LILACS.

A quarta etapa consistiu na categorização dos resultados pela composição de um quadro composto por título do periódico, autores, métodos e ano da publicação. Os objetivos, resultados e discussão, conclusão ou considerações foram apresentados em forma de quadros e tabelas. Na quinta etapa, foram realizadas as interpretações dos artigos e a discussão dos resultados para evidenciar o diagnóstico das cardiopatias congênitas. A sexta etapa consistiu na apresentação da revisão e síntese do conhecimento. Todo o percurso de seleção dos artigos foi descrito no fluxograma (Figura 1). 


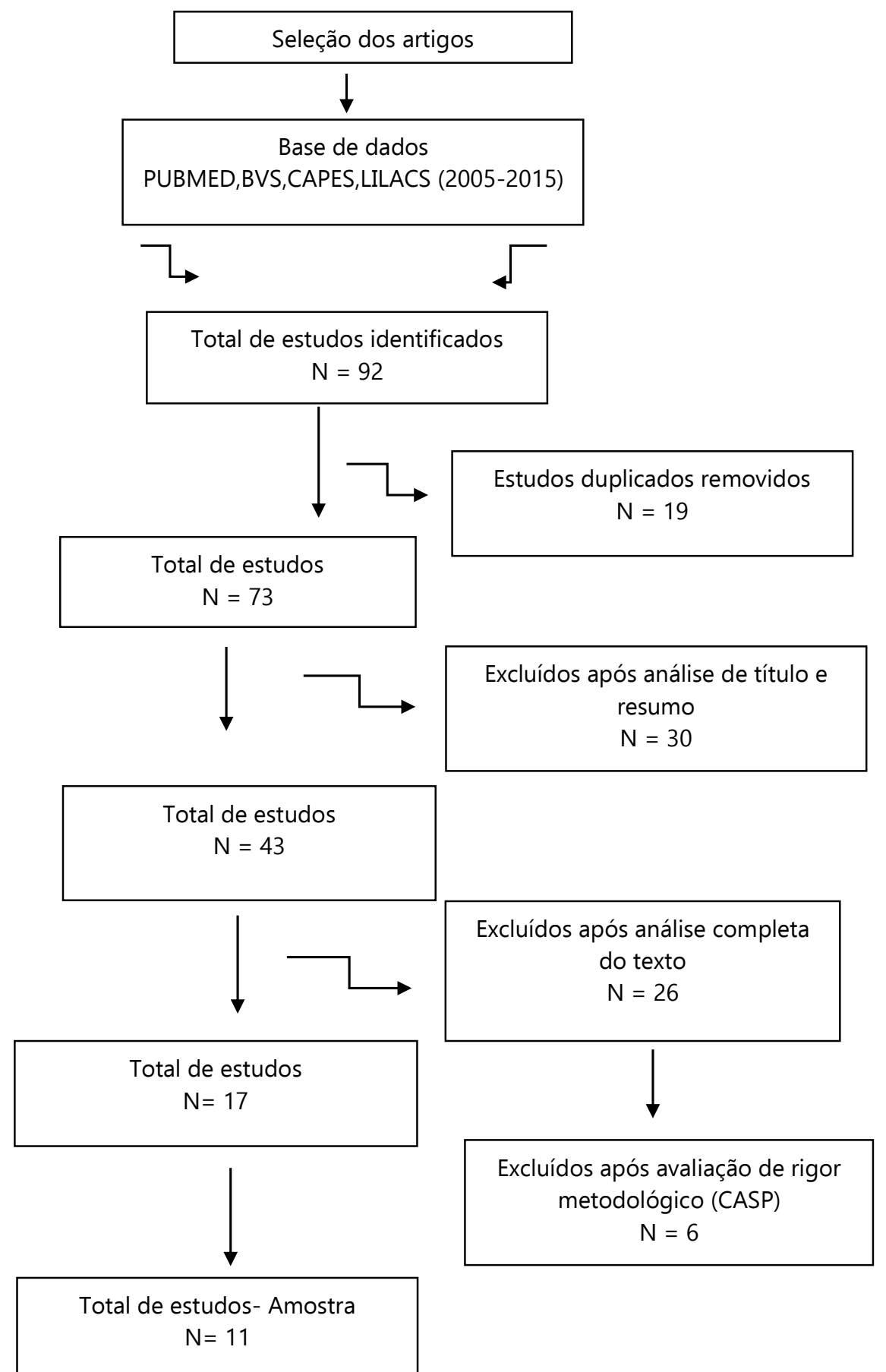

Figura 1. Fluxograma de seleção dos artigos

Fonte: Elaborado pelos autores (2015). 


\section{Resultados}

Em relação à avaliação dos artigos por rigor metodológico da CASP12, segue a distribuição dos 11 artigos selecionados (Quadro 1).

\begin{tabular}{|c|c|}
\hline Número do artigo & Escore segundo CASP \\
\hline 1 & 10 \\
\hline 2 & 10 \\
\hline 3 & 9 \\
\hline 4 & 8 \\
\hline 5 & 8 \\
\hline 6 & 8 \\
\hline 7 & 7 \\
\hline 8 & 7 \\
\hline 9 & 7 \\
\hline 10 & 6 \\
\hline 11 & 6 \\
\hline
\end{tabular}

Quadro 1. Classificação dos artigos por escores segundo a Critical Appraisal Skills Programme CASP. São Luís, 2015.

Fonte: Critical Appraisal Skills Programme (2015).

Foram classificados como de boa qualidade metodológica e viés reduzido 11 artigos, recebendo pontuação de 06 a 10. A tabela 1 a seguir refere-se à distribuição dos artigos segundo a origem, tipo e objetivo do estudo. 
Tabela 1. Distribuição dos artigos segundo a origem, tipo e objetivo do estudo, São Luís, 2015.

\begin{tabular}{|c|c|c|c|}
\hline $\begin{array}{l}\text { Identificação } \\
\text { do artigo }\end{array}$ & Origem & Tipo & Objetivo \\
\hline 1 & Brasil - Ceará & $\begin{array}{l}\text { Estudo } \\
\text { descritivo, } \\
\text { transversal }\end{array}$ & $\begin{array}{c}\text { Conhecer o perfil sociodemográfico familiar e clínico de } \\
\text { crianças com cardiopatia congênita atendidas em uma } \\
\text { instituição hospitalar }\end{array}$ \\
\hline
\end{tabular}

$\begin{array}{ccc}\text { EUA - } & \text { Estudo } \\ \text { Washington, DC } & \text { retrospectivo }\end{array}$

Avaliar a utilidade do ecocardiograma fetal precoce em um programa de cardiologia fetal para a detecção de doença cardíaca congênita em gestações de alto risco

\begin{tabular}{|c|c|c|c|}
\hline 3 & $\begin{array}{c}\text { Brasil - Espírito } \\
\text { Santo }\end{array}$ & $\begin{array}{l}\text { Estudo } \\
\text { retrospectivo } \\
\text { do tipo } \\
\text { validação de } \\
\text { teste } \\
\text { diagnóstico }\end{array}$ & $\begin{array}{c}\text { Avaliar a acurácia da medida da translucência nucal entre } \\
11 \text { e } 13 \text { semanas e } 6 \text { dias de gestação como marcador } \\
\text { ultra-sonográfico para rastreamento cardiopatia } \\
\text { congênita. }\end{array}$ \\
\hline 4 & $\begin{array}{c}\text { Brasil - Ribeirão } \\
\text { Preto }\end{array}$ & $\begin{array}{l}\text { Estudo de } \\
\text { análise } \\
\text { retrospectiva. }\end{array}$ & $\begin{array}{c}\text { Analisar os resultados obtidos em programas de } \\
\text { rastreamento e diagnóstico de arritmias e cardiopatias } \\
\text { congênitas centrado em uma unidade terciária e } \\
\text { determinar a importância do diagnóstico precoce na } \\
\text { evolução fetal e neonatal. }\end{array}$ \\
\hline 5 & Brasil - Alagoas & $\begin{array}{c}\text { Estudo } \\
\text { prospectivo }\end{array}$ & $\begin{array}{c}\text { Analisar a importância dos sintomas, como motivo de } \\
\text { interconsulta com o cardiologista pediátrico, no } \\
\text { diagnóstico de cardiopatias congênitas em recém- } \\
\text { nascidos. }\end{array}$ \\
\hline 6 & $\begin{array}{l}\text { Brasil - Rio } \\
\text { Grande do Sul }\end{array}$ & $\begin{array}{c}\text { Estudo } \\
\text { transversal }\end{array}$ & $\begin{array}{c}\text { Descrever o perfil dos pacientes com cardiopatia } \\
\text { congênita de um serviço de referência no Estado do Rio } \\
\text { Grande do Sul. }\end{array}$ \\
\hline 7 & $\begin{array}{c}\text { Austrália - } \\
\text { Victoria }\end{array}$ & $\begin{array}{l}\text { Estudo } \\
\text { retrospectivo }\end{array}$ & $\begin{array}{l}\text { Determinar a prevalência de cardiopatia congênita em } \\
\text { Victoria- Autrália e avaliar a detecção pré-natal de sete } \\
\text { cardiopatias congênita selecionadas. }\end{array}$ \\
\hline 8 & EUA - Califórnia & $\begin{array}{c}\text { Estudo } \\
\text { transversal }\end{array}$ & $\begin{array}{l}\text { Descrever a distribuição simultânea de saturação de } \\
\text { oxigênio pré e pós-ductal em recém-nascidos } \\
\text { assintomáticos que passaram por uma triagem para a } \\
\text { cardiopatia congênita nas primeiras } 24 \text { horas após o } \\
\text { nascimento. }\end{array}$ \\
\hline 9 & Itália - Di Venero & $\begin{array}{l}\text { Estudo } \\
\text { Retrospectivo }\end{array}$ & $\begin{array}{c}\text { Comparar os achados cardíacos entre } 11 \text { a } 14 \text { semanas } \\
\text { de gestação com os resultados do segundo trimestre } \\
\text { para avaliar a confiabilidade ecocardiografia do primeiro } \\
\text { trimestre e a possibilidade de evolução da doença } \\
\text { cardíaca congênita }\end{array}$ \\
\hline
\end{tabular}


ISSN 2179-6750

\begin{tabular}{ccc} 
Chile - Santiago & $\begin{array}{c}\text { Estudo } \\
\text { descritivo }\end{array}$ & $\begin{array}{c}\text { Comparar a evolução e prognóstico de pacientes com } \\
\text { diagnóstico pré-natal de cardiopatia congênita. }\end{array}$ \\
\hline $\begin{array}{c}\text { Brasil - Rio } \\
\text { Grande do Sul }\end{array}$ & $\begin{array}{c}\text { Estudo } \\
\text { descritivo de } \\
\text { base } \\
\text { populacional }\end{array}$ & $\begin{array}{c}\text { Analisar a frequência de malformações congênitas no } \\
\text { Rio Grande do Sul, enfocando aquelas passíveis de } \\
\text { diagnóstico pré-natal. }\end{array}$
\end{tabular}

Fonte: Elaborado pelos autores (2015).

O maior número de publicações foi de 2006 com 3 artigos, seguido por publicações no ano de 2007, 2010 e 2014, todos com 2 artigos. Seis artigos foram nacionais com destaque o Rio Grande do Sul e 5 artigos internacionais com destaque os Estados Unidos. Identificou-se 5 artigos retrospectivos, 3 artigos descritivos, 2 transversais e 1 prospectivo. A caracterização a seguir refere-se à distribuição dos artigos segundo o autor/ano e diagnóstico das cardiopatias (Tabela 2).

Grande parte dos artigos (7) descrevem que o diagnóstico precoce das cardiopatias congênitas foi realizado por meio da ultrassonografia obstétrica, sendo que 2 apontam a eficácia da ultrassonografia obstétrica juntamente com o ecocardiograma para determinação do diagnóstico. Dois artigos especificaram a realização única do exame de ecocardiograma fetal para o diagnóstico e 2 descrevem a realização de outros exames para o rastreamento diagnóstico, como o exame clínico e a saturação periférica de oxigênio nas primeiras 24 horas. 
Tabela 2. Distribuição dos artigos segundo o autor/ano e diagnóstico das cardiopatias congênitas. São Luís, 2015.

\begin{tabular}{lc}
\hline \multicolumn{1}{c}{ Identificação do artigo } & Diagnóstico das Cardiopatias \\
\hline $\begin{array}{l}\text { Frota MA, Silva IA, Santos } \\
\text { ZMSA, Silva CABS, } \\
\text { Fernandes AFC }\end{array}$ & $\begin{array}{c}\text { O diagnóstico foi determinado pelo exame } \\
\text { ultrassonográfico obstétrico, ainda no período } \\
\text { gestacional }\end{array}$ \\
\hline Tatani, SB & O diagnóstico foi realizado pela ecocardiografia fetal, \\
entre 12 e 16 semanas de gestação
\end{tabular}

Bruns RF, Moron AF, Murta CGV, Gonçalves LFA, Zamith MM

Carvalho SEM, Mendes MC, Cavalli RC, Berezowski
A translucência nucal foi utilizada para o diagnóstico e rastreamento para a cardiopatia congênita

A ultrassonografia obstétrica foi realizada para o rastreamento e o ecocardiograma fetal para o diagnóstico
Identificação do artigo

Guimarães JI

\section{Diagnóstico das Cardiopatias}

O exame físico foi determinante com identificação de presença de sopro, cianose e sinais insuficiência cardíaca após análise do ecocardiograma realizado em recém - nascidos vivos

Bravo-Valenzuela, Nathalie O diagnóstico foi realizado durante o pré-natal por JM, Passarelli MLB, Coates meio de exames como a ultrassonografia obstétrica

VM, Nascimento LFC

A ultrassonografia obstétrica em gestantes com mais de 13 semanas foi capaz de diagnosticar cardiopatias

Pinto Jr, Valdester C congênitas.

A saturação de oxigênio nas primeiras 24 horas dos recém - nascidos foi capaz de diagnosticar

Tandon A, Sengupta S cardiopatias congênitas

Volpe P, Roberts V,

O diagnóstico foi realizado por meio do Campobasso G, Tempesta A, Volpe $G$, Rembouskos $G$ ecocardiograma ainda na fase fetal, no primeiro trimestre de gestação

Aguilera S, Rodríguez JG, Enríquez $\mathrm{G}$

O diagnóstico foi obtido por meio de ecocardiograma após realização de ultrassonografia obstétrica

O estudo morfológico fetal de ultrassonografia pôde diagnosticar precocemente as cardiopatias

Tellles JAB, Faccini LS congênitas.

Fonte: Elaborado pelos autores (2015). 
As CC possuem manifestações clínicas distintas e críticas e por isso, necessitam de diagnóstico nos primeiros dias de vida, devido à rápida evolução para condições ameaçadoras da vida. ${ }^{10} \mathrm{O}$ diagnóstico das cardiopatias pode ser tão precoce como fetal ou tardio como na idade adulta, na dependência do acesso, da disponibilidade tecnológica e profissional ou de sintomas pouco exuberantes que não levem o paciente à procura de tratamento. O cuidado vai do acompanhamento clínico a cateterismo a intervenções cirúrgicas. A depender da complexidade da doença, as intervenções podem ser estagiadas, a que chamamos de paliativas, ou corretivas, nas quais se pretende a resolução do defeito cardíaco. Há, entretanto, necessidade de repetir intervenções motivadas por defeitos residuais, como comunicações entre cavidades, obstruções e incompetências valvares; em outros casos, é necessário substituição de próteses que apresentam disfunção, implantes de marcapassos para tratamento de lesões do sistema de condução, ou até transplante do coração em casos de comprometimento irreversível da função miocárdica. Neste contexto, o planejamento na assistência toma dimensão complexa pela necessidade do diagnóstico precoce, dos cuidados do feto ao adulto, do acompanhamento clínico à cirurgia, da promoção à reabilitação da saúde. ${ }^{8}$

A detecção precoce da anormalidade cardíaca torna-se fundamental e pode evitar intercorrências como choque, acidose, parada cardíaca ou agravos neurológicos, também o conhecimento sobre o perfil da população pode ajudar na elaboração de planos de cuidados e estratégias de intervenção. ${ }^{5}$ O sucesso terapêutico permite um seguimento sistematizado e de qualidade proporcionando o aparecimento de uma nova população de adultos portadores de cardiopatia congênita. Nessa perspectiva, o diagnóstico precoce, especialmente no pré-natal, torna-se fundamental o que contribui para um melhor atendimento do recém-nascido e aumenta as chances de sucesso no tratamento. ${ }^{1,13,14}$

O desafio no estabelecimento de uma rede de atenção à saúde voltada a uma subtemática é delimitar espaço em programas já implementados com o objetivo de agregar soluções para necessidades específicas de uma população com riscos definidos 
pela complexidade das doenças e dos procedimentos terapêuticos. Atuação na atenção primária com programas de prevenção tem influência nas complicações tardias com diminuição nas demandas por intervenções, no plano terciário, que requerem hospitalizações de alto custo. A maior parte das cardiopatias revela sinais e induz a sintomas já na primeira semana de vida. No achado de algum sinal ou sintoma que aponta para o diagnóstico de anormalidades do aparelho cardiocirculatório a busca de acesso ao cardiopediatra, aos exames de imagem para o diagnóstico e à cirurgia cardiovascular no tratamento, expõe limitações. ${ }^{8}$

Há evidência de uma alta taxa de mortalidade intrauterina e pós-natal dcorrente de cardiopatia congênita. Os exames de imagem como a ultrassonografia obstétrica (translucência nucal) e o ecocardiograma fetal, principalmente realizados até o primeiro trimestre foram os mais utilizados para identificação das anormalidades cardíacas, sendo fundamentais para o diagnóstico conclusivo. O exame de saturação periférica de oxigênio e o exame físico criterioso também foram utilizados. O número de doenças congênitas registradas parece estar subestimado e que apesar da maioria das anormalidades serem diagnosticadas no início da gravidez, algumas podem desenvolver-se e tornar-se aparente apenas ao final da gestação ou após o nascimento. O diagnóstico precoce, ainda no prénatal, realizado por profissional capacitado, melhora o prognóstico das crianças.

As anormalidades que atingem a estrutura cardíaca ao nascimento, possuem alto índice de mortalidade, no primeiro ano de vida, amplo espectro clínico com formas sintomáticas e assintomáticas, sendo, portanto, fundamental o diagnóstico precoce. ${ }^{15,16} \mathrm{~A}$ realização de exames diagnósticos, quando precoce e preciso, pode mudar a história da criança, permitindo tratamento adequado e, por vezes, cura definitiva em fase precoce da vida. $^{5}$ O avanço da tecnologia aumentou à sobrevida das crianças portadoras e o diagnóstico das CC pode ser realizado no período gestacional, ao nascimento e durante os primeiros meses de vida. A ultrassonografia obstétrica tem a capacidade de detectar lesões 
de maior gravidade, aumentando as chances de intervenções ainda no período neonatal, evitando complicações e morte. ${ }^{17}$

O poder de detecção de malformações pela ultrassonografia é determinado pela capacidade e conhecimento do profissional. Estudo no Instituto de Cardiologia de Porto Alegre, ${ }^{18}$ identificou-se que $24,7 \%$ das crianças internadas por cardiopatias haviam sido submetidas a avaliação ultrassonográfica no pré-natal após 18 semanas e tiveram suspeita de anormalidades cardíacas. O estudo morfológico fetal poderá diagnosticar precocemente as CC ampliando o diagnóstico pré-natal dessa patologia.

O diagnóstico das CC ainda no pré-natal permite uma avaliação em tempo oportuno e mais acurada no que tange as intervenções necessárias. As cardiopatias congênitas que têm a maior possibilidade de diagnóstico pré-natal são as que apresentam formas mais graves (alteração das quatro câmaras e nas vias de saída) associadas a anomalias cromossômicas e malformações extracardíacas, e, por conseguinte apresentam um risco aumentado de morte neonatal. Os avanços nos métodos diagnósticos, e principalmnete na precocidade de realização dos procedimentos terapêuticos, aumentam as chances da poppulação de indivíduos susceptíveis e consequentemente aumentam a expectativa de vida nesses pacientes. ${ }^{1,19,20}$ A Tabela 3 refere-se à distribuição dos artigos segundo a conclusão e/ou considerações. 
Tabela 3. Distribuição dos artigos segundo conclusão e/ou considerações dos estudos. São Luís, 2015.

Número do artigo Conclusões e/ou Consideraçães
1 Houve predominância do diagnóstico precoce com a utilização prevalecendo à
faixa etária de seis meses, principalmente nas crianças com cardiopatia do tipo
cianótica.

Apesar da baixa sensibilidade, a translucência nucal aumentada é um importante fator de risco para cardiopatias congênitas, devendo ser incluída na estratégia do seu rastreamento pré-natal.

O estudo ultrassonográfico obstétrico é fundamental no rastreamento das alterações cardíacas fetais. O ecocardiograma fetal apresentou alto índice de sensibilidade e especificidade no diagnóstico das arritmias e cardiopatias congênitas, possibilitando o tratamento precoce das alterações graves.

O principal motivo da interconsulta foi à ausculta de sopro, rapidamente detectado no exame físico do recém-nascido, outros foram a presença de cianose e insuficiência cardíaca indicaram alta. Tendo a triagem pediátrica papel importante na detecção dos achados. Ressalta-se a importância do médico pediatra para identificar manifestações precoces da cardiopatia congênita.

$O$ atraso neuropsicomotor e o baixo ganho pôndero-estatural podem estar associados às cardiopatias congênitas. Indica novas questões de pesquisa para 6 discutir a etiologia e a incidência crescente das malformações cardíacas.

O diagnóstico pré-natal de cardiopatias congênitas melhora o prognóstico das crianças como a ultrassonografia para triagem dos defeitos congênitos. Sugerese a inclusão de profissional habilitado para a realização da ultrassonografia pré-

7 natal.

A importância do uso da oximetria periférica nas primeiras 24 horas de vida do recém-nascido para avaliar a saturação de oxigênio torna-se essencial para a detecção e rastreio de cardiopatias congênitas.

O ecocardiograma fetal realizado no $1^{\circ}$ trimestre parece permitir consideravelmente a detecção precoce de doença cardíaca congênita grave. Embora a maioria das formas de defeitos cardíacos congênitos possa ser diagnosticada no início da gravidez, alguns podem desenvolver e tornar-se aparente apenas mais tarde na gestação ou após o nascimento.

A importância do diagnóstico pré-natal com o ecocardiograma mostra a evidência que existe uma alta taxa de mortalidade tanto intra-uterino e pósnatal decorrente de cardiopatia congênita e, por conseguinte, de difícil manejo com diminuição de taxa de sobrevivência. Há evidência de que crianças que possuem o diagnóstico ainda no pré-natal teriam um menor tempo de cirurgia e de internação em unidades de traratamento intensivo, com possibilidade de melhorar a morbidade e desenvolvimento neurológico a longo prazo. 
O número de doenças congênitas registradas parece estar subestimado para alguns defeitos congênitos, especialmente aqueles cujos diagnósticos necessitam de exames especializados, como as cardiopatias congênitas.

Fonte: Elaborado pelos autores (2015).

A translucência nucal é um componente a ser avaliado no momento da ulltrassonografia, extremamente importante para o rastreamento das cardiopatias congênitas realizada entre a $11^{a}$ a $13^{a}$ semana de gravidez. Deve-se sempre suspeitar de cardiopatia congênita quando é detectado um aumento do líquido na região da nuca do feto, principalmente na presença de um cariótipo normal. Da mesma forma, todo feto com cariótipo alterado deve ter seu coração avaliado por especialista, visto que as cardiopatias são extremamente frequentes nesse grupo. A translucência nucal mostra-se como um excelente marcador para encaminhameto de exame ecocardiográfico detalhado. ${ }^{21}$

A ultrassonografia consiste na avaliação de todos os órgãos e sistemas, enfantizando-se o sistema cardiovascular, mediante a obtenção de cortes básicos (quatro câmaras, eixos longos e eixos curtos) e determinação da frequencia e rítmo cardíaco, estabelecendo alterações nestes sem necessariamente definir o tipo de malformação. Já o ecocardiograma é especializado na avaliação cardíaca fetal, abordando exclusivamete o sistema cardiovascular, com obtenção de corte transverso (para determinar a posição da aorta e veia cava inferior em relação a coluna vertebral), um corte das câmaras cardíacas para avaliação das cavidades cardíacas, e, da junção atrioventricular, além de cortes de eixo longo e eixo curto que demonstram a relação entre átrios e ventrículos, estabelecendo o diagnóstico específico para malformações cardíacas. ${ }^{22,23}$

Estudo comparou a utilização a ultrassonografia e ecocardiograma ${ }^{22}$ e identificou uma taxa de sucesso superior do ecocardiograma (75\%) em comparação a ultrassonografia obstétrica (72\%). Quando o ecocardiograma é realizado no pré-natal existe a possibilidade de tratamento ainda intra-utero o que demonstra a importância do rastreamento e da indicação do ecocardiograma fetal no diagóstico específico das cardiopatias. Entretanto, 
apesar do ecocardiograma ser o método ideal para o diagnóstico de CC, seja fetal ou pósnatal, a sua utilização como ferramenta de triagem parece inviável (custo e escassez de profissionais). O ecocardiograma fetal é conveniente para aprofundar a investigação sempre que a ultrassonografia sugerir algum defeito na formação do coração fetal, uma vez que podem haver associações e pode ser realizado a partir do primeiro trimestre da gestação, comumente entre 18 a 22 semanas de idade gestacional. A indicação mais frequente para o ecocardiograma fetal ocorre em mulheres com história familiar significativa de doença cardíaca congênita ou em gravidez com doença cardíaca préexistente. $^{23,24}$

O diagnóstico pré-natal por meio do ecocardiograma fetal reduz a mortalidade por essa doença, entretanto para isso, deve-se utilizar outros métodos de triagem, como o exame clínico criterioso e a oximetria de pulso. A adoção de modelos de triagem para cardiopatias congênitas torna-se essencial frente ao cenário atual e constituem o resultado do trabalho conjunto de uma equipe multidisciplinar. ${ }^{10,19} \mathrm{O}$ exame clínico criterioso no recém-nascido pode determinar manifestações precoces de cardiopatias congênitas que não foram observadas em exames pré-natais como a ausculta de sopro cardíaco, presença de cianose, desconforto respiratório e outros sinais que podem passar despercebidos durante o exame físico. ${ }^{25}$

A morbimortalidade das cardiopatias congênitas, assim como o processo do seu diagnóstico, é um problema mundial. Isso se torna ainda mais grave em países subdesenvolvidos, onde o déficit de profissionais capacitados e dificuldades estruturais nos serviços de saúde são uma constante. Adicionalmente, o fato de os cardiologistas pediátricos estarem concentrados nos grandes centros urbanos resulta num grande número de neonatos descobertos. Nesse contexto, a adição de competências para outros profissionais é essencial para uma triagem adequada no contexto atual. A oximetria de pulso arterial é um bom método de triagem e que pode, inclusive, ser realizado por profissionais de enfermagem. Dessa forma, nesse fluxo diagnóstico, a inserção da 
enfermagem para realização da oximetria e do neonatologista para realização de ecocardiograma no recém-nascido é vantajosa. Os neonatologistas além de terem mais experiência com o exame físico e processos fisiopatológicos nos neonatos, também são responsáveis pelas altas hospitalares dos mesmos. Logo, a capacitação de tais profissionais para realização da oximetria de pulso arterial facilmente realizada e do ecocardiograma com apoio de cardiologistas pediátricos só tem a adicionar. ${ }^{10}$

A aferição da oximetria de pulso é uma tarefa bastante simples, sendo necessário que o recém-nascido esteja com as extremidades aquecidas (membro superior ou inferior) e o monitor evidencie uma onda de traçado homogêneo. O resultado normal consiste na saturação periférica maior ou igual a 95\% em ambas as medidas e diferença menor que $3 \%$ entre as medidas do membro superior direito e membro inferior. Para resultado anormal, uma nova aferição deverá ser realizada após 1 hora. Caso o resultado se confirme, um ecocardiograma deverá ser realizado dentro das 24 horas. ${ }^{25}$ Este teste apresenta sensibilidade de $75 \%$ e especificidade de 99\%, sendo assim, algumas cardiopatias críticas podem não ser detectadas por ele, principalmente a coarctação de aorta. A realização da oximetria não descarta a necessidade de realização de exame físico minucioso e detalhado em todo recém-nascido, antes da alta hospitalar. ${ }^{26}$

Atualmente, o Sistema Único de Saúde - SUS só disponibiliza o ultrassom quando o feto apresenta algum tipo de má-formação ou quando a gestante possui diabetes, cardiopatia, hipertensão arterial, lúpus ou tem mais de 35 anos de idade. Infelizmente, a disponibilidade de realização de exames diagnósticos para as cardiopatias congênitas no âmbito do SUS é muito abaixo das necessidades da população brasileira e o custo e outros fatores operacionais impactam diretamente na rede de saúde instalada que não é suficiente, alertando para a necessidade do sistema de saúde se preparar para diagnosticar e tratar mais precocemente as cardiopatias congênitas, reduzindo o mau seguimento e acompanhamento, com consequências graves para as possíveis crianças e famílias. 
A capacitação da rede primária com vistas a diagnosticar precocemente as CC, já que não se pode evitar o nascimento desses bebês, a menos que se preconize a interrupção da gestação, desencadeia um processo de cuidados que exige estruturas tecnologicamente densas voltadas para a confirmação diagnóstica, programação de tratamento e intervenções cirúrgicas. Requer também um processo bem definido de contrarreferência para o cuidado desses bebês. A profissão e as demandas sociais instigaram o estudo sobre o tema saúde integral-cuidado-cardiopatias-congênitas. Em vista disso, vem o entendimento da conveniência de criar um modelo de Rede de Atenção Integral à Saúde dos Portadores de Cardiopatias Congênitas. A proposta provoca os governos a tomarem decisões em prol dos que nascem com doença no coração. Passa a ser decisivo pensar na integralidade da atenção e, portanto, no encadeamento de intervenções que possam solucionar demandas financeiras, da gestão, estrutura física, pessoas, tecnologias, logística e integração entre os serviços. Enseja-se trabalhar na direção da integralidade no cuidado, servindo-se das redes de atenção à saúde por meio dos seus elementos constitutivos: a população, a estrutura operacional, o modelo de atenção à saúde e o sistema de avaliação. ${ }^{8}$

Como limitações do estudo ressalta-se a escassez de publicações de base populacional abrangendo amostras maiores e diversificadas e estudos controlados randomizados para avaliar a eficácia dos diagnósticos estabelecidos com ênfase na comparação sistemática entre as populações sobre os critérios e modelos de triagem nos diversos cenários do sistema de saúde.

\section{Considerações Finais}

O diagnóstico das cardiopatias congênitas existe, porém há dificuldade na realização do mesmo de maneira precoce, constituindo uma tarefa difícil por diversos fatores, dentre eles: a variedade e gravidade das cardiopatias, a complexidade das estruturas anatômicas e funcionais, o tipo de exame diagnóstico solicitado, o tempo de 
realização do exame e a categoria profissional envolvida. Sabe-se que o coração dever ser rastreado sistematicamente, pois na maioria das vezes as doenças que ocorrem em fetos não apresentam quaisquer fatores de risco, podendo as cardiopatias serem subdiagnosticadas, subnotificadas e consequentemente subtratadas.

Os métodos diagnósticos mais frequentemente utilizados e com relevância para a sobrevida dos pacientes portadores de cardiopatias congênitas são a ultrassonografia fetal (translucência nucal) e o ecocardiograma fetal. O rastreamento ultrassonográfico na gestante constitui a melhor forma de detecção destas malformações no primeiro trimestre e o ecocardiograma fetal, um método importante utilizado para análise acurada das cardiopatias. Outros métodos utilizados para o rastreamento e diagnóstico das CC nos recém-nascidos são o exame físico criterioso, a oximetria de pulso e o ecocardiograma do recém-nascido, constituindo métodos diagnósticos de rastreamentos eficientes. A avaliação do recém-nascido com suspeita de cardiopatia deve ser realizada de maneira criteriosa e rotineira, nessa perspectiva, destaca-se a inclusão de outros profissionais devidamente treinados para a realização de exames, como a oximetria de pulso arterial (realizada pela enfermagem) e ecocardiograma do recém-nascido (realizado por neonatologistas).

O diagnóstico precoce das CC ainda no pré-natal permite uma avaliação em tempo oportuno e mais acurada no que tange as intervenções necessárias, constata-se a importância do diagnóstico precoce para um tratamento adequado, prevenção de agravos e sequelas oportunizando a melhora do prognóstico e permitindo a sobrevida prolongada à saúde da criança, com ênfase na participação de outros profissionais para a qualidade do resultado final obtido. A descrição de como se dá o diagnóstico das cardiopatias congênitas, torna-se relevante para o planejamento de ações que envolvam a promoção da saúde, prevenção e controle de agravos, oferecendo a oportunidade para ações proativas e elaboração de estratégias apropriadas de assistência, configurando-se, portanto, como uma auditoria da qualidade da atenção à saúde. 


\section{Referências}

1. Huber J, Peres VC, Santos TJ, Beltrão LF, Baumont AC, Canedo AD, et al. Cardiopatias congênitas em um serviço de referência: evolução clínica e doenças associadas. Arq Bras Cardiol. 2010;94(3):333-38. http://dx.doi.org/10.1590/S0066-782X2010000300009.

2. Santos ADS, Menezes GA, Sousa DS. Perfil dos recém-nascidos com cardiopatia congênita em uma maternidade de alto risco do município de Aracaju. Cad Graduac. 2013;1(17):59-70.

3. Bocchi EA, Braga FGM, Ferreira SMA, Rohde LE, Oliveira WA, Almeida DR, et al. III Diretriz da Sociedade Brasileira de Cardiologia para Gravidez na Mulher Portadora de Cardiopatia. Arq Bras Cardiol 2009;93(1 Supl 1):110-78. http://dx.doi.org/10.1590/S0066-782X2009002000001

4. Urakawa IT, Kobayashi RM. Identificação do perfil e diagnóstico de enfermagem do neonato com cardiopatia congênita. Rev Pesq Cuid Fundam. 2012;4(4):3118-24. 10.9789/2175-5361.2012.v4i4.3118-3124.

5. Bastos LF, Araújo TM, Frota N M. Caetano JÁ. Perfil clínico e epidemiológico de crianças com cardiopatias congênitas submetidas à cirurgia cardíaca. Rev Enferm UFPE On Line. 2013;7(8):5298-304. 10.5205/reuol.3452-28790-4-ED.0708201330.

6. Araújo JSS, Régis CT, Gomes RGS, Silva CS, Abath CMB, Mourato FA, et al. Cardiopatia congênita no Nordeste brasileiro: 10 anos consecutivos registrados no Estado da Paraíba, Brasil. Rev Bras Cardiol. 2014;27(1):13-9.

7. Anshula T, Sengupta S, Shukla V, Danda S. Risk factors for Congenital Heart Disease (CHD) in Vellore, India. Curr Res J. 2010;2(4):253-8.

8. Pinto Jr, Valdester C. Cardiopatias congênitas: rede de atenção à saúde. Fortaleza: Edição do autor; 2015.152 p.

9. Mellander M, Sunnegardh J. Failure to diagnose critical heart malformations in newborns before discharge an increasing problem? Acta Paediatric. 2006;95(4):407-13. http://dx.doi.org/10.1080/08035250500541910.

10. Moser LRDN, Diógenes TCP, Souza VOP, Oliveira ARF, Mourato FA, Mattos SS. Novo modelo de teletriagem das cardiopatias congênitas. J Bras Tele. 2014;3(1):229-31. https://doi.org/10.12957/jbrastele.2014.10236.

11. Mendes MJFL, Alves JGB, Alves AV, Siqueira PP, Freire EF. Associação de fatores de risco para doenças cardiovasculares em adolescentes e seus pais. Rev Bras Saude Matern Infant. 2006;6(1):549-54.

12. Critical Appraisal Skills Programme (CASP). Making sense of evidence. Milton Keynes Primary Care Trust, 2006. 
13. Amaral F, Manso PH, Granzotti JA, Vicente WVA, Schmidt A. Cardiopatia congênita no adulto: perfil clínico ambulatorial no Hospital das Clínicas de Ribeirão Preto. Arq Bras Cardiol. 2010;94(6):707-13. http://dx.doi.org/10.1590/S0066-782X2010005000053.

14. Mendes KDS, Silveira RCCP, Galvão CM. Revisão integrativa: método de pesquisa para a incorporação de evidências na saúde e na enfermagem. Texto Contexto Enferm. 2008;17(4):758-64. http://dx.doi.org/10.1590/S0104-07072008000400018.

15. Brasil. Lei no. 9.610, de 19 de fevereiro de 1998. Altera, atualiza e consolida a legislação sobre direitos autorais e dá outras providências. Diário Oficial da União, Brasília, DF; 20 fev. 1998 [citado em 12 fev. 2015]. Disponível em http:// www.dou.gov.br/materias/do1 / do1legleg19980220180939-001. htm.

16. Nordon DG, Prigenzi ML. Cardiopatia congenital: difícil diagnóstico diferencial e condução do tratamento. Rev Fac Cienc Med Sorocaba. 2012;14(1):24-6.

17. Frota MA, Silva IA, Santos ZMSA, Silva CABS, Fernandes AFC. Perfil sociodemográfico familiar e clínico de crianças com cardiopatia congênita atendidas em uma instituição hospitalar. Rev Bras Promoc Saude. 2014,27(2):239-46. http://dx.doi.org/1010.5020/18061230.2014.p239.

18. Tellles JAB, Scüler-Faccini LS Defeitos congênitos no Rio Grande do Sul: diagnóstico ultrassonográfico pelo estudo morfológico fetal. Rev AMRIGS. 2010;54(4):421-26.

19. Aguilera S, Rodríguez JG, Enríquez G. Cardiopatías congénitas con diagnóstico prenatal: seguimiento. Rev Chil Obstet Ginecol. 2006;71(5):320-6. http://dx.doi.org/10.4067/S0717-75262006000500005.

20. Chew C, Halliday JL, Riley MM, Peny DJ. Population-based study of antenatal detection of congenital heart disease by ultrasound examination. Ultrasound Obstet Gynecol. 2007;29(6):619-24. http://dx.doi.org/10.1002/uog.4023.

21. Bruns RF, Moron AF, Murta CGV, Gonçalves LFA, Zamith MM. O papel da translucência nucal no rastreamento de cardiopatias congênitas. Arq Bras Cardiol. 2006;87(3):307-14. http://dx.doi.org/10.1590/S0066-782X2006001600013.

22. Carvalho SEM, Mendes MC, Cavalli RC, Machado JC, Duarte G, Berezowski A. Rastreamento e diagnóstico ecocardiográfico das arritmias e cardiopatias congênitas fetais. Rev Bras Ginecol Obstet. 2006;28(5):304-9. http://dx.doi.org/10.1590/S010072032006000500007

23. Volpe P, Robertis V, Campobasso G, Tempesta A, Volpe G, Rembouskos G. Diagnosis of congenital heart disease by early and second-trimester fetal echocardiography. J Ultrasound Med. 2012;31(4):563-8. https://doi.org/10.7863/jum.2012.31.4.563.

24. Pike JI, Krishnan A, Donofrio MT. Early fetal echocardiography: congenital heart disease detection and diagnostic accuracy in the hands of an experienced fetal cardiology program. Prenat Diagn. 2014;34(8):790-6. https://doi.org/10.1002/pd.4372. 
25. Jegatheesan P, Dongli S, Cathy A, Kamakshi D, Govindaswami B. Oxygen saturation nomogram in newborns screened for critical congenital heart disease. Pediatrics. 2013;131(6):e1803-10. https://doi.org/10.1542/peds.2012-3320.

26. Kemper AR, Mahle WT, Martin GR, Cooley C, Kumar P, Morrow R, et al. Strategies for implementing screening for critical congenital heart disease. Pediatrics. 2011;128(5): e1259-67. https://doi.org/10.1542/peds.2011-1317.

\section{Minicurrículo}

Taynar da Costa Almeida Pavão | ORCiD: 0000-0001-9998-1782

Enfermeira. Especialista em Cardiologia.

Joseildes Castelo Branco Souza | ORCiD: 0000-0001-5351-3393

Enfermeira. Mestre em Ciências da Saúde.

Luzinéa de Maria Pastor Frias | ORCiD: 0000-0003-3493-3207

Enfermeira. Doutora. Docente da Universidade Federal do Maranhão - UFMA.

Líscia Divana Carvalho Silva | ORCiD: 0000-0002-3624-6446

Enfermeira. Doutora. Docente da Universidade Federal do Maranhão - UFMA. 